\title{
Leptogenesis in the Neutrino Option
}

\author{
I. Brivio, ${ }^{a}$ K. Moffat, ${ }^{b}$ S. Pascoli, ${ }^{b}$ S.T. Petcov ${ }^{c, d}$ and J. Turner ${ }^{e}$ \\ ${ }^{a}$ Institut für Theoretische Physik, Universität Heidelberg, \\ Philosophenweg 16, 69120 Heidelberg, Germany \\ ${ }^{b}$ Institute for Particle Physics Phenomenology, Department of Physics, Durham University, \\ South Road, Durham DH1 3LE, United Kingdom \\ ${ }^{c} S I S S A / I N F N$, \\ Via Bonomea 265, I-34136 Trieste, Italy \\ ${ }^{d}$ Kavli IPMU (WPI), University of Tokyo, \\ 5-1-5 Kashiwanoha, 277-8583 Kashiwa, Japan \\ e Theoretical Physics Department, Fermi National Accelerator Laboratory, \\ P.O. Box 500, Batavia, IL 60510, U.S.A. \\ E-mail: brivio@thphys.uni-heidelberg.de, \\ kristian.p.moffat@durham.ac.uk, silvia.pascoli@durham.ac.uk, \\ petcov@sissa.it, jturner@fnal.gov
}

ABstract: We examine the compatibility between the Neutrino Option, in which the electroweak scale is generated by $\mathrm{PeV}$ mass type I seesaw Majorana neutrinos, and leptogenesis. We find the Neutrino Option is consistent with resonant leptogenesis. Working within the minimal seesaw scenario with two heavy Majorana neutrinos $N_{1,2}$, which form a pseudoDirac pair, we explore the viable parameter space. We find that the Neutrino Option and successful leptogenesis are compatible in the cases of a neutrino mass spectrum with normal (inverted) ordering for $1.2 \times 10^{6}<M(\mathrm{GeV})<8.8 \times 10^{6}\left(2.4 \times 10^{6}<M(\mathrm{GeV})<7.4 \times 10^{6}\right)$, with $M=\left(M_{1}+M_{2}\right) / 2$ and $M_{1,2}$ the masses of $N_{1,2}$. Successful leptogenesis requires that $\Delta M / M \equiv\left(M_{2}-M_{1}\right) / M \sim 10^{-8}$. We further show that leptogenesis can produce the baryon asymmetry of the Universe within the Neutrino Option scenario when the requisite $\mathrm{CP}$ violation in leptogenesis is provided exclusively by the Dirac or Majorana low energy $\mathrm{CP}$ violation phases of the PMNS matrix.

KeYwords: Cosmology of Theories beyond the SM, Neutrino Physics, Beyond Standard Model, CP violation

ARXIV EPRINT: 1905.12642 


\section{Contents}

1 Introduction 1

2 The Neutrino Option and leptogenesis 2

2.1 Neutrino masses and mixing 2

2.2 The Neutrino Option 5

2.3 The framework of leptogenesis 6

2.4 Leptogenesis at the scales required for the Neutrino Option 8

3 Results $\quad \mathbf{1 0}$

3.1 Lower bound on the heavy Majorana neutrino masses 10

$\begin{array}{lll}3.2 & \text { Upper bound on the heavy Majorana neutrino masses } & 15\end{array}$

3.3 Leptogenesis from purely low energy CP violation in the Neutrino Option 15

4 Conclusions $\quad 18$

\section{Introduction}

Neutrino oscillation experiments have provided overwhelming evidence for very small but non-zero neutrino masses - a fact that is not explained in the Standard Model (SM). A minimal extension of the SM which addresses this issue is the type I seesaw framework where a number of heavy Majorana neutrinos are added to the SM particle spectrum [1-4]. The light neutrinos have a mass scale inversely proportional to that of the heavy Majorana neutrinos and so their extreme smallness may be naturally explained. In particular, if the type I seesaw mechanism is to generate at least three non-degenerate light neutrino masses, as is required by the data from oscillation experiments, then there must be at least two heavy Majorana neutrinos.

The decays of the heavy Majorana neutrinos in the early Universe may produce the observed matter-antimatter asymmetry in leptogenesis processes [5] (see also, e.g., [6-9]). Lepton-number- and C- and CP-violating decays of the heavy Majorana neutrinos, which generate a lepton asymmetry, may occur out-of-equilibrium thereby satisfying Sakharov's conditions for a dynamical generation of the (leptonic) matter-antimatter asymmetry of the Universe [10]. This lepton asymmetry is subsequently partially processed into a baryon asymmetry by $B+L$-violating sphaleron processes.

However, there exists a tension between leptogenesis within the type I seesaw mechanism and the naturalness of the Higgs potential. This is because radiative corrections to the Higgs potential increase monotonically with the mass scale of the heavy Majorana neutrinos. In a natural scenario, where the corrections to the Higgs mass do not exceed $1 \mathrm{TeV}$, the heavy Majorana neutrino mass scale must satisfy $M<3 \times 10^{7} \mathrm{GeV}[11,12]$, 
which is considerably lower than the Davidson-Ibarra bound for successful leptogenesis $M \gtrsim 10^{9} \mathrm{GeV}^{1}[13-15]$.

A different perspective on this problem is brought by the so-called Neutrino Option scenario $[16,17]$ which is based on the idea that the Higgs potential is generated by the radiative corrections of the heavy Majorana neutrinos, starting from an approximately conformal scalar potential at the seesaw scale. In this framework the heavy Majorana neutrino masses are the only dimensionful parameters of the theory and they control both the breaking of the conformal symmetry and that of lepton number.

In this work we shall investigate the possibility of successful leptogenesis within the Neutrino Option framework and focus on the minimal scenario where there are only two heavy Majorana neutrinos providing both the Higgs mass and the baryon asymmetry. We show that in order for leptogenesis to be successful within the Neutrino Option approach to electroweak symmetry breaking, it is necessary for the two heavy neutrinos to be close in mass (forming a pseudo-Dirac pair $[18,19]$ ) and their masses to be in the range $M \sim 10^{6}-10^{7} \mathrm{GeV}$. From these considerations, we derive an upper and lower bound on this mass scale. The upper bound coincides with that found for the Neutrino Option alone [17], while the lower bound comes from the additional requirement of viable leptogenesis. We explore the naturalness in the neutrino sector in terms of the presence of an approximately conserved lepton charge. We investigate also the production of the baryon asymmetry when the requisite $\mathrm{CP}$ violation in leptogenesis is provided exclusively by the low energy phases of the PMNS matrix.

\section{The Neutrino Option and leptogenesis}

In this section we review neutrino masses in the type I seesaw (section 2.1). We then summarise the essential features of the Neutrino Option in section 2.2 before reviewing the relevant features of leptogenesis in section 2.3. Finally in section 2.4 we consider which leptogenesis scenarios may be viable in conjunction with the Neutrino Option.

\subsection{Neutrino masses and mixing}

Neutrino oscillation experiments have shown that neutrinos have small but non-zero masses and that they mix. The mass and flavour states of neutrinos are not aligned and their misalignment is described by the PMNS matrix $U$ :

$$
\nu_{\alpha L}=\sum_{i=1}^{3} U_{\alpha i} \nu_{i L}
$$

where $\nu_{\alpha L}$ is the flavour neutrino field, $\alpha \in\{e, \mu, \tau\}$, and $\nu_{i}$ is the field of the neutrino of mass $m_{i}$. We make use of the conventional PDG parametrisation [20]:

$$
U=\left(\begin{array}{ccc}
c_{12} c_{13} & s_{12} c_{13} & s_{13} e^{-i \delta} \\
-s_{12} c_{23}-c_{12} s_{23} s_{13} e^{i \delta} & c_{12} c_{23}-s_{12} s_{23} s_{13} e^{i \delta} & s_{23} c_{13} \\
s_{12} s_{23}-c_{12} c_{23} s_{13} e^{i \delta} & -c_{12} s_{23}-s_{12} c_{23} s_{13} e^{i \delta} & c_{23} c_{13}
\end{array}\right)\left(\begin{array}{ccc}
1 & 0 & 0 \\
0 & e^{i \frac{\alpha_{21}}{2}} & 0 \\
0 & 0 & e^{i \frac{\alpha_{31}}{2}}
\end{array}\right),
$$

\footnotetext{
${ }^{1}$ We recall that the Davidson-Ibarra bound is valid for hierarchical heavy Majorana neutrino mass spectrum and in the case of absence of flavour effects in leptogenesis.
} 


\begin{tabular}{|cccccc|}
\hline $\begin{array}{c}\theta_{13} \\
\left(^{\circ}\right.\end{array}$ & $\begin{array}{c}\theta_{12} \\
\left(^{\circ}\right)\end{array}$ & $\begin{array}{c}\theta_{23} \\
\left(^{\circ}\right.\end{array}$ & $\begin{array}{c}\delta \\
\left(^{\circ}\right.\end{array}$ & $\begin{array}{c}\Delta m_{21}^{2} \\
\left(10^{-5} \mathrm{eV}^{2}\right)\end{array}$ & $\begin{array}{c}\Delta m_{3 l}^{2} \\
\left(10^{-3} \mathrm{eV}^{2}\right)\end{array}$ \\
\hline $8.61_{-0.13}^{+0.12}$ & $33.82_{-0.76}^{+0.78}$ & $49.7_{-1.1}^{+0.9}$ & $217_{-28}^{+40}$ & $7.39_{-0.20}^{+0.21}$ & $2.525_{-0.031}^{+0.033}$ \\
\hline $8.65_{-0.13}^{+0.12}$ & $33.82_{-0.75}^{+0.78}$ & $49.7_{-1.0}^{+0.9}$ & $280_{-28}^{+25}$ & $7.39_{-0.20}^{+0.21}$ & $-2.512_{-0.031}^{+0.034}$ \\
\hline
\end{tabular}

Table 1. Best fit value and $1 \sigma$ ranges of the neutrino oscillation parameters from a global fit to neutrino data [22]. Upper (lower) values are for neutrino mass spectrum with normal (inverted) ordering, for which $\Delta m_{3 l}^{2} \equiv \Delta m_{31}^{2}\left(\Delta m_{3 l}^{2} \equiv \Delta m_{32}^{2}\right)$.

where $c_{i j} \equiv \cos \theta_{i j}, s_{i j} \equiv \sin \theta_{i j}, \delta$ is the Dirac phase and $\alpha_{21}, \alpha_{31}$ are the Majorana phases [21]. In addition to the mixing angles and phases, neutrino oscillation experiments provide precise measurements of the two independent neutrino mass squared differences $\Delta m_{21}^{2}$ and $\Delta m_{31(32)}^{2}$. The best-fit values of $\theta_{12}, \theta_{23}, \theta_{13}, \delta, \Delta m_{21}^{2}$ and $\Delta m_{31(32)}^{2}$, obtained in one of the most recent analyses of the global neutrino oscillation data [22], are given in table 1 . The three neutrino masses $m_{1}, m_{2}$ and $m_{3}$ may be arranged into two possible orderings compatible with the oscillation data: normal ordering (NO) for $m_{1}<m_{2}<m_{3}$; and inverted ordering (IO) $m_{3}<m_{1}<m_{2}$. Although the mass ordering has not yet been determined experimentally, there is a mild statistical preference for normal ordering in the data $[22]$.

A simple means of explaining the smallness of neutrino masses is the type I seesaw framework [1-4], where heavy Majorana neutrinos, $N_{i}(i \in\{1,2,3, \ldots\})$, are added to the SM spectrum. After electroweak symmetry is broken, when the Higgs has developed a vacuum expectation value (vev) $v \approx 174 \mathrm{GeV}$, the neutrino mass terms of the Lagrangian are given by

$$
\mathcal{L}_{m}=-\frac{1}{2}\left(\bar{\nu}_{L}, \bar{N}_{L}^{c}\right)\left(\begin{array}{cc}
0 & v Y \\
v Y^{T} & M_{R}
\end{array}\right)\left(\begin{array}{c}
\nu_{R}^{c} \\
N_{R}
\end{array}\right)+\text { h.c. }
$$

where $\nu_{L}^{T} \equiv\left(\nu_{e L}^{T}, \nu_{\mu L}^{T}, \nu_{\tau L}^{T}\right)$ and $N_{R}^{T} \equiv\left(N_{1 R}^{T}, N_{2 R}^{T}, N_{3 R}^{T}, \ldots\right)$ are the flavour neutrino fields and the fields of the heavy Majorana mass eigenstates respectively and $Y$ is the matrix of the neutrino Yukawa couplings of the heavy Majorana neutrinos $N_{i}$ to the leptonic and Higgs doublets. In eq. (2.3), $\left.\left(\nu_{R}^{c}\right)^{T}=\left(\left(\nu_{e R}^{c}\right)^{T},\left(\nu_{\mu R}^{c}\right)^{T},\left(\nu_{\tau R}^{c}\right)^{T}\right)\right),\left(N_{L}^{c}\right)^{T}=$ $\left(\left(N_{1 L}^{c}\right)^{T},\left(N_{2 L}^{c}\right)^{T},\left(N_{3 L}^{c}\right)^{T}, \ldots\right)$, with $\nu_{l R}^{c}=C\left(\bar{\nu}_{l L}\right)^{T}, l \in\{e, \mu, \tau\}$, and $N_{j L}^{c}=C\left(\bar{N}_{j R}\right)^{T}$ for $j \in\{1,2,3, \ldots\}$, where $C$ denotes the charge conjugation matrix. We work in a basis in which the Majorana mass matrix $M_{R}$ is diagonal $M_{R}=\operatorname{diag}\left(M_{1}, M_{2}, \ldots\right)$.

The light neutrino mass matrix, $m_{\nu}$, may be brought to a semi-positive diagonal form (denoted by a caret) using the Takagi transformation:

$$
\hat{m}_{\nu}=U^{\dagger} m_{\nu} U^{*}
$$

such that $\hat{m}_{\nu}=\operatorname{diag}\left(m_{1}, m_{2}, m_{3}\right)$, with $m_{i}$ the mass corresponding to $\nu_{i}$.

By analogy with the method of Casas and Ibarra [23], we apply the seesaw formula for light neutrino masses

$$
m_{\nu} \approx-v^{2} Y M_{R}^{-1} Y^{T}
$$


to parametrise the Yukawa matrix as

$$
Y=\frac{1}{v} U \sqrt{\hat{m}_{\nu}} R^{T} \sqrt{M_{R}}
$$

where $R$ is a $3 \times 3$ complex orthogonal matrix. Here we drop a phase factor of $i$, which would cancel from all our results. We note that this result, which is correct at tree-level, will suffice in this work as radiative corrections to the light neutrino masses will be negligible.

In what follows we consider the case in which only two heavy Majorana neutrinos are present, or equivalently, the case when the third heavy Majorana neutrino, $N_{3}$, decouples. This is the minimal scenario compatible with the oscillation data where one of the three light neutrinos is massless. For concreteness, we also assume $M_{1} \leq M_{2}$. In the considered scenario, the sum of neutrino masses is $\sum_{i=1}^{3} m_{\nu_{i}} \cong 0.058(0.010) \mathrm{eV}$ for NO (IO) neutrino mass spectrum, which is well within the cosmological upper limit reported by the Planck collaboration, $\sum_{i} m_{i}<0.120-0.160 \mathrm{eV}$ at $95 \%$ C.L. [24].

The assumption of $N_{3}$ decoupling implies that we may take $m_{1}=0$ for the NO spectrum (at tree-level). Correspondingly we have for the $R$-matrix:

$$
R=\left(\begin{array}{ccc}
0 & \cos \theta & \sin \theta \\
0 & -\sin \theta & \cos \theta \\
1 & 0 & 0
\end{array}\right)
$$

where $\theta=x+i y$. This in turn leads to

$$
U^{\dagger} Y=\frac{1}{v}\left(\begin{array}{cc}
0 & 0 \\
\sqrt{M_{1}} \sqrt{m_{2}} \cos (x+i y) & -\sqrt{M_{2}} \sqrt{m_{2}} \sin (x+i y) \\
\sqrt{M_{1}} \sqrt{m_{3}} \sin (x+i y) & \sqrt{M_{2}} \sqrt{m_{3}} \cos (x+i y)
\end{array}\right) .
$$

Similarly, for IO spectrum we have $m_{3}=0$ and subsequently

$$
R=\left(\begin{array}{ccc}
\cos \theta & \sin \theta & 0 \\
-\sin \theta & \cos \theta & 0 \\
0 & 0 & 1
\end{array}\right)
$$

In this case

$$
U^{\dagger} Y=\frac{1}{v}\left(\begin{array}{cc}
\sqrt{M_{1}} \sqrt{m_{1}} \cos (x+i y) & -\sqrt{M_{2}} \sqrt{m_{1}} \sin (x+i y) \\
\sqrt{M_{1}} \sqrt{m_{2}} \sin (x+i y) & \sqrt{M_{2}} \sqrt{m_{2}} \cos (x+i y) \\
0 & 0
\end{array}\right)
$$

In the literature a phase factor $\xi= \pm 1$ is sometimes included in the definition of $R$ to allow for both the cases $\operatorname{det}(R)= \pm 1$. We have chosen instead to extend the range of the Majorana phases $\alpha_{21(31)}$ to $[0,4 \pi]$ such that the same full set of possible Yukawa matrices has been considered [25]. 


\subsection{The Neutrino Option}

It has been recently suggested that the heavy Majorana states, $N_{i}$, introduced in the type I seesaw model could be responsible for the dynamical generation of the scalar potential of the Standard Model, in addition to that of neutrino masses $[16,17]$. In this scenario, dubbed the Neutrino Option, the classical potential is given by

$$
V_{0}(\Phi)=-\frac{M_{H 0}^{2}}{2} \Phi^{\dagger} \Phi+\lambda_{0}\left(\Phi^{\dagger} \Phi\right)^{2}
$$

and is assumed to be nearly conformal at the seesaw scale: $M_{H 0}(\mu \gtrsim M) \simeq 0, \lambda_{0}(\mu \gtrsim M) \neq 0$, $\mu$ being the renormalisation scale. Radiative corrections to both $M_{H}^{2}$ and $\lambda$ are generated via the diagrams of figure 1 , thus breaking scale invariance at the quantum level. At energies $\mu<M$ the $N_{i}$ fields can be integrated out and decoupled from the spectrum. One is then left with an Effective Field Theory (EFT) in which the leading seesaw contributions are encoded in the Weinberg operator for neutrino masses (stemming from the seesaw-EFT matching at tree-level) and in finite threshold matching contributions to the Higgs potential parameters $\Delta M_{H}^{2}, \Delta \lambda$ (stemming from the one-loop matching). The latter are identified as the zeroth order term in the $E / M$ expansion of the loops, computed with dimensional regularisation within the $\overline{M S}$ renormalisation scheme. For the case of two heavy Majorana neutrinos with $M_{2}=x_{M} M_{1}, x_{M} \geq 1$ they read [17]:

$$
\begin{aligned}
\Delta M_{H}^{2}= & \frac{M_{1}^{2}}{8 \pi^{2}}\left(\left|Y_{1}\right|^{2}+x_{M}^{2}\left|Y_{2}\right|^{2}\right) \\
\Delta \lambda= & -\frac{1}{32 \pi^{2}}\left[5\left|Y_{1}\right|^{4}+5\left|Y_{2}\right|^{4}+2 \operatorname{Re}\left(Y_{1} \cdot Y_{2}^{*}\right)^{2}\left(1-\frac{2 \log x_{M}^{2}}{1-x_{M}}\right)\right. \\
& \left.+2 \operatorname{Im}\left(Y_{1} \cdot Y_{2}^{*}\right)^{2}\left(1-\frac{2 \log x_{M}^{2}}{1+x_{M}}\right)\right],
\end{aligned}
$$

with $Y_{i}$ the $i$ th column of the matrix of neutrino Yukawa couplings. In the limit $M_{2}=M_{1}$ $\left(x_{M}=1\right)$ these reduce to: ${ }^{2}$

$$
\begin{aligned}
\Delta M_{H}^{2} & =\frac{M_{1}^{2}}{8 \pi^{2}}\left(\left|Y_{1}\right|^{2}+\left|Y_{2}\right|^{2}\right), \\
\Delta \lambda & =-\frac{5}{32 \pi^{2}}\left(\left|Y_{1}\right|^{4}+\left|Y_{2}\right|^{4}+2 \operatorname{Re}\left(Y_{1} \cdot Y_{2}^{*}\right)^{2}\right)-\frac{1}{16 \pi^{2}} \operatorname{Im}\left(Y_{1} \cdot Y_{2}^{*}\right)^{2} .
\end{aligned}
$$

The values of $m_{H}, \lambda$ at the EW scale can be extrapolated using the renormalisation group equations (RGEs) of the SM [26] (as the heavy neutrinos are not present in the spectrum) with the following boundary conditions:

$$
M_{H}^{2}\left(\mu=M_{1}\right) \equiv \Delta M_{H}^{2} \quad \text { and } \quad \lambda\left(\mu=M_{1}\right) \equiv \Delta \lambda+\lambda_{0} .
$$

The condition on $M_{H}$ places the strongest constraint on the parameter space of the Neutrino Option, requiring $M_{i} \lesssim 10^{7} \mathrm{GeV}$ and $\left|Y_{\alpha i}\right| \sim 1 \mathrm{TeV} / M_{i}$ (barring tunings between the

\footnotetext{
${ }^{2}$ Note that although modified Feynman rules must be used when the heavy Majorana neutrinos are nearly degenerate in mass, this is only important when results, such as the CP asymmetry, depend on the difference of masses. In the calculation of the Higgs mass parameter, the contributions from each heavy Majorana neutrino are summed and any correction depending on the difference of the masses is negligible.
} 

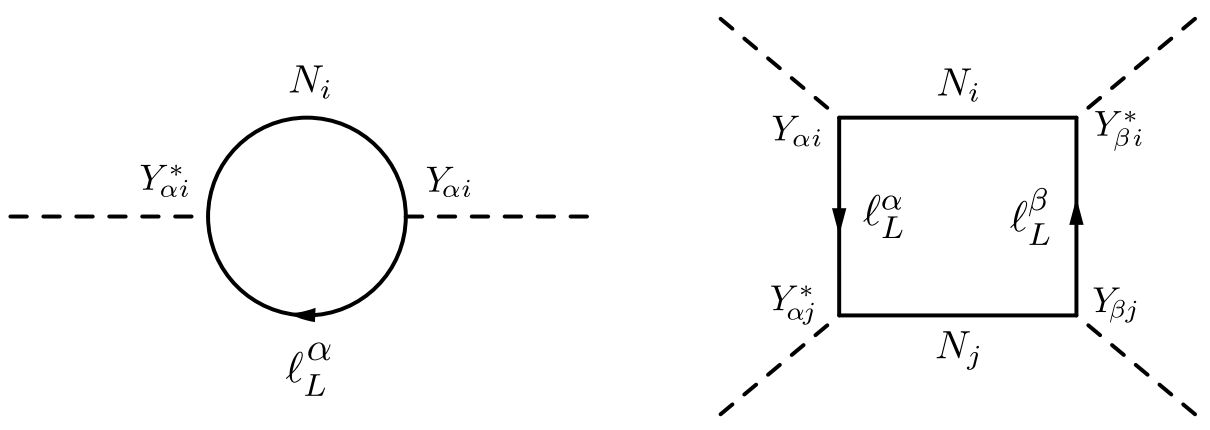

Figure 1. The dominant one-loop contribution generating the Higgs potential in the type-I seesaw model. Here $\ell_{L}^{\alpha}$ denotes the $\mathrm{SU}(2)$ lepton doublet, as the EW symmetry is unbroken at this stage.

Yukawa entries) in order to reproduce the correct Higgs mass and, at the same time, be consistent with the constraints from neutrino oscillation experiments. As neither $M_{H}$ nor the light neutrino masses change significantly under RGE running, this result is consistent to a good approximation with the order-of-magnitude estimate

$$
M_{H}^{2}(\mu=v) \simeq \Delta M_{H}^{2} \sim \frac{M_{i}^{2}\left|Y_{i}\right|^{2}}{8 \pi^{2}} \quad \text { and } \quad m_{\nu}(\mu=v) \simeq \frac{v^{2}\left|Y_{i}\right|^{2}}{2 M_{i}} .
$$

Within this region of the parameter space the contribution to the Higgs quartic term is $|\Delta \lambda| \leq 10^{-7}$. As a consequence, the threshold matching contribution is always negligible in comparison to the coupling in the classical potential which has to be positive and of loop size $\left(\lambda_{0} \simeq 0.01-0.05\right)$ in order to obtain the correct scalar potential at the EW scale [17].

\subsection{The framework of leptogenesis}

The type I seesaw provides a possible mechanism for the generation of the matter-antimatter asymmetry if the heavy Majorana neutrinos $N_{i}$ decay out-of-equilibrium, in C-/CP- and lepton-number-violating processes in the early Universe. These decays produce a leptonic matter-antimatter asymmetry which may be transformed in part into the observed baryon asymmetry by SM sphaleron processes which violate the $B+L$ charge but conserve the $B-L$ one. Any such process which generates a lepton asymmetry that is then converted into a baryon asymmetry is called leptogenesis.

The baryon-to-photon ratio, which parametrises the baryon asymmetry of the Universe (BAU), is defined as

$$
\eta_{B} \equiv \frac{n_{B}-n_{\bar{B}}}{n_{\gamma}}
$$

where $n_{B}, n_{\bar{B}}$ and $n_{\gamma}$ are the number densities of baryons, antibaryons and photons respectively. Its value has been inferred by two independent methods: the measurement from Big-Bang nucleosynthesis (BBN), which occurs when the temperature of the Universe satisfies $T \lesssim 1 \mathrm{MeV}$ [27], and the measurement of $\eta_{B}$ from Cosmic Microwave Background radiation $(\mathrm{CMB})$ data $[28]$, which probes it at the time of recombination, $T \lesssim 1 \mathrm{eV}$. As the latter value has been more precisely measured, we choose to use it throughout:

$$
\eta_{B \mathrm{CMB}}=(6.02-6.18) \times 10^{-10},
$$

where the above is the $3 \sigma$ range of the measurement. 
The Boltzmann equations for thermal leptogenesis provide a semi-classical description of the time evolution of the heavy neutrino densities, $n_{N_{i}}(i \in\{1,2,3, \ldots\})$ with equilibrium distributions $n_{N_{i}}^{\text {eq }}$, and the lepton asymmetry, $n_{B-L} \cdot{ }^{3}$ At the scales $T \lesssim 10^{7} \mathrm{GeV}$, at which the Neutrino Option can be viable, the flavour effects in leptogenesis are important. Each flavour component of the leptons produced in $N_{i}$ decays feels a different interaction with the plasma of the early Universe and decoheres from the other two. Consequently, the kinetic equations must separately describe the time evolution of the asymmetries in the tauon, muon and electron lepton charges, $n_{\tau \tau}, n_{\mu \mu}, n_{e e}$. The total baryon asymmetry is computed by taking the sum $n_{B-L}=n_{\tau \tau}+n_{\mu \mu}+n_{e e}$ and multiplying it by a factor such that $\eta_{B} \approx 0.01 n_{B-L}$. In this regime all three lepton flavour asymmetries $n_{\tau \tau}, n_{\mu \mu}$ and $n_{e e}$ evolve independently and the Boltzmann equations are given by:

$$
\begin{aligned}
& \frac{d n_{N_{i}}}{d z}=-D_{i}\left(n_{N_{i}}-n_{N_{i}}^{\mathrm{eq}}\right), \\
& \frac{d n_{\alpha \alpha}}{d z}=\sum_{i}\left(\epsilon_{\alpha \alpha}^{(i)} D_{i}\left(n_{N_{i}}-n_{N_{i}}^{\mathrm{eq}}\right)-p_{i \alpha} W_{i} n_{\alpha \alpha}\right), \quad i=1,2, \alpha=e, \mu, \tau .
\end{aligned}
$$

where $z \equiv M_{1} / T$ and increases with time, $p_{i \alpha}$ and $\bar{p}_{i \alpha}$ are the projection probabilities from decay state $i$ to the flavour state $\alpha$ for particles and antiparticles, respectively, and $\epsilon_{\alpha \alpha}^{(i)}$ is the CP asymmetry in the lepton charge $\alpha$ generated in the decay of the heavy Majorana neutrino $N_{i}$. The projection probabilities are given by

$$
\begin{aligned}
& p_{i \alpha} \equiv\left|c_{i \alpha}\right|^{2}, \\
& \bar{p}_{i \alpha} \equiv\left|\bar{c}_{i \alpha}\right|^{2},
\end{aligned}
$$

where $c_{i \alpha}$ and $\bar{c}_{i \alpha}$ are the projection coefficients of the charged lepton products of the decay of the $i$ th heavy Majorana neutrino $\left(N_{i} \rightarrow \bar{\Phi} l_{i}, N_{i} \rightarrow \Phi \bar{l}_{i}\right)$ in

$$
\begin{aligned}
& \left|\ell_{i}\right\rangle=\sum_{\alpha} c_{i \alpha}\left|l_{\alpha}\right\rangle, \\
& \left|\bar{\ell}_{i}\right\rangle=\sum_{\alpha} \bar{c}_{i \alpha}\left|\bar{l}_{\alpha}\right\rangle,
\end{aligned}
$$

and $\epsilon_{\alpha \alpha}^{(1)}$ the CP asymmetry (given in eq. (2.22)) with $i=1,2,3$ and $\alpha=e, \mu, \tau$. For further details we refer the reader to ref. [29].

The decay parameter, $D_{i}$, describes the decay of $N_{i}$ and is defined in terms of the heavy neutrino decay rate $\Gamma_{i} \equiv \Gamma_{i}\left(N_{i} \rightarrow \bar{\Phi} \ell_{i}\right)$ with $\Phi$ the Higgs and the CP-conjugate rate, $\bar{\Gamma}_{i} \equiv \Gamma_{i}\left(N_{i} \rightarrow \Phi \bar{\ell}_{i}\right)$, and Hubble rate, $H[7]$ :

$$
D_{i} \equiv \frac{\Gamma_{i}+\bar{\Gamma}_{i}}{H z} .
$$

Likewise, the washout factor is defined in terms of the heavy neutrino inverse decay rate $\Gamma_{i}^{\mathrm{ID}} \equiv \Gamma_{i}\left(\bar{\Phi} \ell_{i} \rightarrow N_{i}\right)$ and the CP-conjugate inverse decay rate $\bar{\Gamma}_{i}^{\mathrm{ID}} \equiv \Gamma_{i}\left(\Phi \bar{\ell}_{i} \rightarrow N_{i}\right)$ is

$$
W_{i} \equiv \frac{1}{2} \frac{\Gamma_{i}^{\mathrm{ID}}+\bar{\Gamma}_{i}^{\mathrm{ID}}}{H z} .
$$

\footnotetext{
${ }^{3}$ All number densities are normalised to a volume containing a single heavy Majorana neutrino in ultrarelativistic thermal equilibrium.
} 
We also define the washout-parameter

$$
K_{i} \equiv \frac{\tilde{m}_{i}}{m_{*}}, \quad \text { where } \quad \tilde{m}_{i} \equiv \frac{\left(Y^{\dagger} Y\right)_{i i} v^{2}}{M_{i}}
$$

and $m_{*}=\left(16 \pi^{2} v^{2} / 3 M_{P}\right) \sqrt{\left(g_{*} \pi\right) / 5} \approx 10^{-3} \mathrm{eV}$ with $M_{P}$ the Planck mass.

As will be discussed in further detail in section 2.4, we find it necessary to take the two heavy Majorana neutrinos to be nearly-degenerate in mass $\Delta M=M_{2}-M_{1} \ll M \equiv$ $\frac{1}{2}\left(M_{1}+M_{2}\right)$ and thereby $\Delta M \sim \Gamma$. In this case, one is concerned with resonant leptogenesis $[9,30]$ where the self-energy contribution to the CP asymmetry parameter may become large. Such enhancement of the asymmetry can be significant, allowing the energy scale for successful leptogenesis to be lowered by several orders of magnitude. For this reason, resonant leptogenesis has been most often explored in the literature within scenarios with Majorana masses of the order of a few $\mathrm{TeV}$. Here we apply this paradigm to a wider energy range. The peculiarity of resonant leptogenesis is that non-negligible contributions to the CP-asymmetry can be induced by mixing and oscillation of the heavy Majorana neutrinos. The mixing effects come from the possibility of off-diagonal transitions in the self-energy diagrams at $T=0$ which are included through use of the resummed Yukawa couplings [31]. In the same regime, $\Delta M \sim \Gamma$, the thermal contributions to the self-energies are also important. This provides an extra contribution to the $\mathrm{CP}$ asymmetries in processes where on-shell heavy Majorana neutrinos oscillate in flavour space due to their interactions with a thermal background [32]. The CP asymmetry which takes account of both the mixing and oscillation of the heavy Majorana neutrinos has the form [33]:

$$
\epsilon_{\alpha \alpha}^{(i)}=\sum_{j \neq i} \frac{\operatorname{Im}\left[Y_{i \alpha}^{\dagger} Y_{\alpha j}\left(Y^{\dagger} Y\right)_{i j}\right]+\frac{M_{i}}{M_{j}} \operatorname{Im}\left[Y_{i \alpha}^{\dagger} Y_{\alpha j}\left(Y^{\dagger} Y\right)_{j i}\right]}{\left(Y^{\dagger} Y\right)_{i i}\left(Y^{\dagger} Y\right)_{j j}}\left(f_{i j}^{\mathrm{mix}}+f_{i j}^{\mathrm{osc}}\right)
$$

where

$$
f_{i j}^{\operatorname{mix}}=\frac{\left(M_{i}^{2}-M_{j}^{2}\right) M_{i} \Gamma_{j}}{\left(M_{i}^{2}-M_{j}^{2}\right)^{2}+M_{i}^{2} \Gamma_{j}^{2}}
$$

and

$$
f_{i j}^{\text {osc }}=\frac{\left(M_{i}^{2}-M_{j}^{2}\right) M_{i} \Gamma_{j}}{\left(M_{i}^{2}-M_{j}^{2}\right)^{2}+\left(M_{i} \Gamma_{i}+M_{j} \Gamma_{j}\right)^{2} \frac{\operatorname{det}\left[\operatorname{Re}\left(Y^{\dagger} Y\right)\right]}{\left(Y^{\dagger} Y\right)_{i i}\left(Y^{\dagger} Y\right)_{j j}}} .
$$

\subsection{Leptogenesis at the scales required for the Neutrino Option}

The largest value of the heavy Majorana neutrino masses compatible with the Standard Model Higgs mass in the Neutrino Option scenario is $M_{i} \sim 10^{7} \mathrm{GeV}$. A lower bound on the heavy Majorana neutrino masses can be set by the requirement of perturbativity of the neutrino Yukawa couplings and is $M_{i} \gtrsim \mathcal{O}\left(10^{2}\right) \mathrm{GeV} .{ }^{4}$ In such a regime, in order for the

\footnotetext{
${ }^{4}$ The lower bound on $M_{1}$ as shown in figure (3) of [17] comes from the additional assumption that $|\sin (x+i y)|<1$, that constrains the width of the allowed bands in the figure. This assumption was introduced in order to forbid explicitly fine tunings in the flavor space, but can be relaxed in full generality.
} 
neutrino masses to satisfy the existing limits, fine tuned cancellations must exist between the tree level seesaw and the one-loop contributions to the light neutrino masses.

From the lower bound derived from perturbativity arguments to the upper bound from the Neutrino Option itself, there are two possible mechanisms of leptogenesis viable in the relevant heavy Majorana neutrino mass range:

1. Thermal leptogenesis with enhanced $R$-matrices [34] (see also [35, 36]).

2. Resonant leptogenesis with nearly degenerate heavy Majorana neutrino masses [30, 37-44].

In this subsection, we shall argue that the fine-tuned scenario detailed in (1) is incompatible with the Neutrino Option, and therefore justify our exclusive use the resonant method for the investigations in this work. The arguments we present here are valid in the more general case of three heavy Majorana neutrinos which we consider below.

In the fine-tuned case the elements of the $R$-matrix elements tend to be large and the one-loop contribution to the light neutrino masses should be incorporated through modification of the Casas-Ibarra parametrisation [45]. In this case, the $R$-matrix has the structure [34]:

$$
R \approx\left(\begin{array}{ccc}
R_{11} & R_{12} & R_{13} \\
\pm i R_{22} & R_{22} & R_{23} \\
-R_{22} & \pm i R_{22} & \pm i R_{23}
\end{array}\right)
$$

Here $\left|R_{22}\right| \gg\left|R_{1 i}\right|,\left|R_{23}\right|$ for $i \in\{1,2,3\}$, which results, in [34]

$$
m^{\text {tree }} \approx-m^{1 \text {-loop }} .
$$

A typical fine-tuned leptogenesis solution, which allows for partial cancellations between the tree and one-loop level contributions to the light neutrino masses, requires $M_{1} \approx 5 \times 10^{6} \mathrm{GeV}$ (see the scenarios of [34]) and correspond to large values $\sqrt{\Delta M_{H}^{2}} \sim 8 \times 10^{6} \mathrm{GeV}$. The latter value owes its magnitude to the dependence of $\Delta M_{H}^{2}$ on the $R$-matrix elements which are themselves large in order to provide the fine-tuning of eq. (2.26).

We performed a numerical search of the parameter space with heavy neutrino masses $\sim 10^{6} \mathrm{GeV}$ and found no points which simultaneously satisfy the requirements of the Neutrino Option and leptogenesis even when we allowed $m^{\text {tree }}$ and $m^{1-\text { loop }}$ to cancel to $\sim 0.1 \%$. When no constraint was placed on the levels of fine-tuning it was possible to find solutions with the desired value of $\eta_{B}$ and $\sqrt{\Delta M_{H}^{2}} \sim 100 \mathrm{GeV}$. Such solutions corresponds to an $R$-matrix with very large entries, $\left|R_{i j}\right| \sim 10^{12}$, and very small (physically unreasonable) heavy neutrino masses $M_{i} \sim 10^{-2} \mathrm{GeV}$. The fine-tuned cancellation between the tree- and one-loop light neutrino masses is so complete that the higher-order radiative corrections to the light neutrino masses dominate and exceed the light neutrino mass bound. Using the estimate that the two-loop contribution to the light neutrino masses is

$$
\left|m^{\text {two-loop }}\right| \sim \frac{1}{16 \pi^{2}}\left|m^{\text {one-loop }}\right| \max .(|Y|)^{2} \approx \frac{1}{16 \pi^{2}}\left|m^{\text {tree }}\right| \max .(|Y|)^{2},
$$


where max. $(|Y|)$ is the largest element of the matrix of absolute values of neutrino Yukawa couplings, and where we use the approximate equality of eq. (2.26), we estimate

$$
m^{\text {two-loop }} \sim 10^{3} \mathrm{GeV},
$$

which is well-excluded by the experimental constraints. In summary, we found only physically non-viable solutions involving fine-tuned non-resonant leptogenesis. For this reason, for the remainder of this work we restrict ourselves to resonant leptogenesis in which $M_{1} \approx M_{2}$ and where $N_{3}$ is decoupled.

\section{Results}

In this section we present our main results. In section 3.1, we derive the lower bound on the mass scale $M$ for which leptogenesis is viable within the Neutrino Option and explore the available parameter space and in section 3.2 we find the corresponding upper bound. In section 3.3 we find the conditions under which it is possible to achieve successful leptogenesis in the Neutrino Option when the CP violation comes purely from the low-energy phases of the PMNS matrix.

\subsection{Lower bound on the heavy Majorana neutrino masses}

In this section we determine the range of heavy Majorana neutrino masses in which both the Neutrino Option and leptogenesis are viable. We shall always work in the nearly degenerate case $\Delta M \equiv M_{2}-M_{1} \ll M$ where $M \equiv\left(M_{1}+M_{2}\right) / 2$. Evaluating $\Delta M_{H}^{2}$ at $\mu \sim M$ as is calculated in [17], the threshold correction is

$$
\Delta M_{H}^{2}=\frac{1}{8 \pi^{2}} \operatorname{Tr}\left[Y M^{2} Y^{\dagger}\right] .
$$

By substitution of the Casas-Ibarra parametrisation of eq. (2.8) or eq. (2.10), this becomes

$$
\Delta M_{H}^{2}=\frac{1}{8 \pi^{2} v^{2}} \cosh (2 y) M^{3}\left(m_{1}+m_{2}+m_{3}\right),
$$

where $m_{1}=0\left(m_{3}=0\right)$ for NO (IO) spectrum and the neutrino parameters run with the scale M. This effect amounts to a few percent for the light neutrino masses and is implemented here using the RGEs of refs. [46, 47]. The remainder of the neutrino parameters change less significantly when RG evolved, so their scale dependence will be neglected. Note that the $x$ parameter (the real part of $\theta$ which parametrises the $R$ matrix), cancels in (3.2) due to the near-diagonality of the heavy Majorana mass matrix. The Neutrino Option is then satisfied if the Standard Model $\overline{M S}$ Higgs mass, when renormalisation group evolved to the scale $M$, matches the values given by $\Delta M_{H}^{2}$. The running of the SM parameters is taken into account implementing the RGE of ref. [26] to the highest available accuracy and with the numerical inputs reported in table 2.

The lower bound on $M$ for leptogenesis in the Neutrino Option is the lowest scale for which the correct baryon asymmetry results whilst still satisfying eq. (3.2). We apply the approximate analytical solution

$$
n_{B-L} \approx \frac{\pi^{2}}{6 z_{d}} n^{\mathrm{eq}}(0) \sum_{\alpha=e}^{\tau} \frac{\epsilon_{\alpha \alpha}^{(1)}}{K_{1} p_{1 \alpha}}
$$




\begin{tabular}{|lc|}
\hline inputs & value $(\mathrm{GeV})$ \\
\hline$v$ & 174.10 \\
$M_{H}$ & 125.09 \\
$M_{t}$ & 173.2 \\
\hline
\end{tabular}

\begin{tabular}{|lclc|}
\hline \multicolumn{4}{|c|}{ RGE boundary conditions at $\mu=M_{t}$} \\
\hline$\lambda$ & 0.1258 & $M_{H}(\mathrm{GeV})$ & 131.431 \\
$g_{1}$ & 0.461 & $Y_{t}$ & 0.933 \\
$g_{2}$ & 0.644 & $Y_{b}$ & 0.024 \\
$g_{3}$ & 1.22029 & $Y_{\tau}$ & 0.0102 \\
\hline
\end{tabular}

Table 2. Values of the relevant SM parameters adopted in the numerical analysis, consistent with ref. [17]. The RGE boundary conditions are computed at the highest accuracy provided in ref. [26].

in the derivation of which we have eliminated $\epsilon_{\alpha \alpha}^{(2)}$ with the approximation $\epsilon_{\alpha \alpha}^{(2)} / K_{2} p_{2 \alpha} \approx$ $\epsilon_{\alpha \alpha}^{(1)} / K_{1} p_{1 \alpha}$. For the lowest heavy Majorana neutrino mass scale $M$, the value of $y$ (the imaginary part of $\theta$ ) will be largest as can be seen from inspection of eq. (3.2). Approximating the terms in the sum under the assumption that $e^{y} \gg e^{-y}$ (to be justified later), we find

$$
\begin{aligned}
& \frac{\epsilon_{\alpha \alpha}^{(1)}}{K_{1} p_{1 \alpha}} \approx 16 m_{*}\left(f_{\text {osc }}+f_{\text {mix }}\right) \frac{m_{2}-m_{3}}{\left(m_{2}+m_{3}\right)^{2}} e^{-4 y} \sin 2 x, \text { Normal Ordering }, \\
& \frac{\epsilon_{\alpha \alpha}^{(1)}}{K_{1} p_{1 \alpha}} \approx 16 m_{*}\left(f_{\text {osc }}+f_{\text {mix }}\right) \frac{m_{1}-m_{2}}{\left(m_{1}+m_{2}\right)^{2}} e^{-4 y} \sin 2 x, \quad \text { Inverted Ordering. }
\end{aligned}
$$

Using the same approximation, the factor $\propto \operatorname{det}\left[\operatorname{Re}\left(Y^{\dagger} Y\right)\right]$ in the denominator of $f_{\text {osc }}$ (see eq. (2.24)) becomes

$$
\frac{\operatorname{det}\left[\operatorname{Re}\left(Y^{\dagger} Y\right)\right]}{\left(Y^{\dagger} Y\right)_{11}\left(Y^{\dagger} Y\right)_{22}} \approx 1
$$

From eq. (3.4) we observe that the parameter $y$ exponentially suppresses the final asymmetry $\eta_{B}$ while enhancing the Higgs mass of eq. (3.2). To the level of accuracy in the approximation, the contribution of each flavour is identical. Although the left-hand side carries a flavour index $\alpha$, the right-hand side is independent of this index. As the flavour information is contained in the PMNS elements, we expect that at large $y$, the solutions for successful leptogenesis in the Neutrino Option should have only a weak dependence on the PMNS phases (in terms that have been neglected in eq. (3.4)).

The lower bound on $M$ may be found by maximising the terms in eq. (3.4) with respect to all parameters except $y$ and then finding the largest value of $y$ for which leptogenesis may be successful. From eq. (3.2), we observe the scale of $M$ is determined from $y$ (recalling that the light neutrino masses are to be run to the scale $M$ ) and may therefore infer the lower bound for successful leptogenesis in the Neutrino Option. The maximisation of the right-hand side of eq. (3.4) occurs when $x=135^{\circ}$ or $315^{\circ}$ and $\Delta M / \Gamma_{2} \approx 0.61$ as shown in figure 2. We find that the values of $y$ that give agreement with $\eta_{B_{\mathrm{CMB}}}$ are $y=190.22^{\circ}$ for normal ordering and $y=118.21^{\circ}$ for inverted ordering. These imply lower bounds for viable leptogenesis in the Neutrino Option of

$$
\begin{array}{ll}
M>1.2 \times 10^{6} \mathrm{GeV} & \text { Normal Ordering, } \\
M>2.4 \times 10^{6} \mathrm{GeV} & \text { Inverted Ordering. }
\end{array}
$$




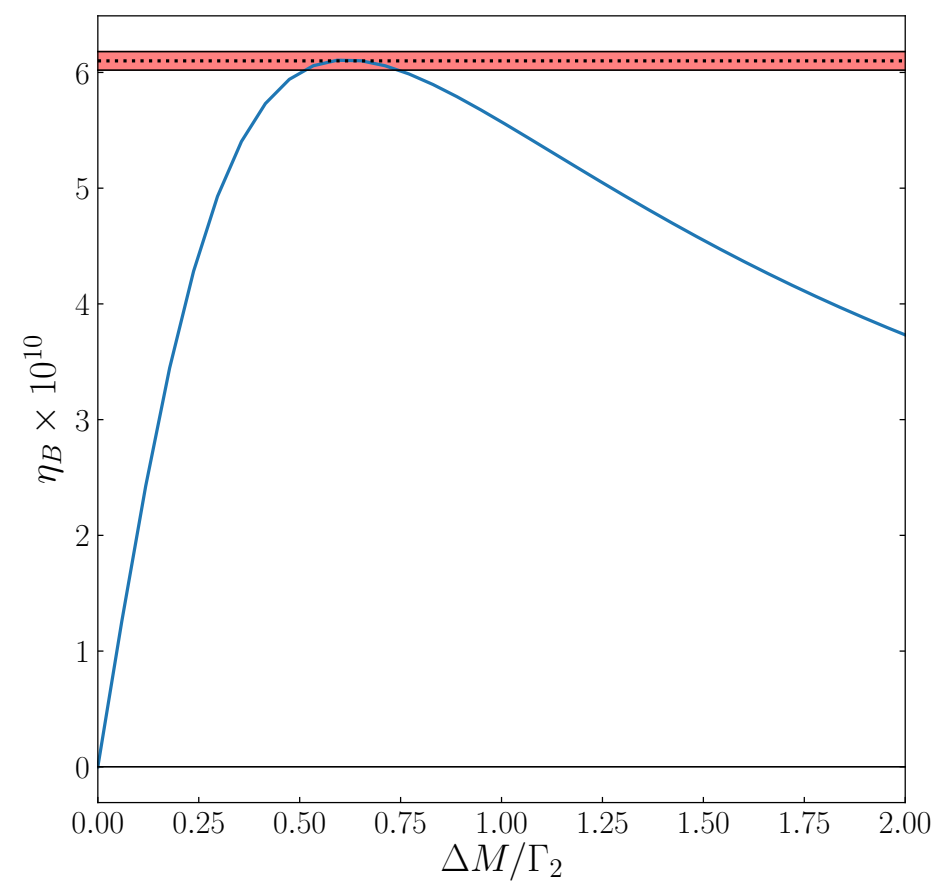

Figure 2. The baryon asymmetry as a function of the heavy Majorana neutrino mass splitting divided by the decay rate of $N_{2}$ at the lower-bound of $M$ for successful leptogenesis in the Neutrino Option. The plots take an identical form for both normal ordering (for which $\Gamma_{2}=1.62 \times 10^{-2} \mathrm{GeV}$ ) and inverted ordering (for which $\Gamma_{2}=8.63 \times 10^{-3} \mathrm{GeV}$ ).

The difference in values for the two bounds is entirely determined by the difference in the factors $\left(m_{2}-m_{3}\right) /\left(m_{2}+m_{3}\right)^{2}$ and $\left(m_{1}-m_{2}\right) /\left(m_{1}+m_{2}\right)^{2}$ appearing for normal and inverted ordering respectively. We emphasise that the suppression factor $e^{-4 y}$ occurring in eq. (3.4) is sufficiently strong that the lower bounds are not strongly affected by the running of the parameters (although the bounds stated include the running of all SM parameters and the light neutrino masses).

As a cross-check we have also determined the lower bound on $M$ for which leptogenesis is viable within the Neutrino Option by numerically solving the resonant Boltzmann equations (we stress we solve the Boltzmann equations and not the analytically approximated equations of eq. (3.4)) for both normal and inverted ordering and scanned the available parameter space for $\eta_{B}=6.1 \times 10^{-10}$. We performed the parameter space exploration using Multinest [48-50] for a fixed scale $M$ but varied the splitting $\Delta M$, with $y$ fixed to the value that satisfies $M_{H}^{2}\left(M_{1}\right)=\Delta M_{H}^{2}\left(M_{1}\right)$. That is, a value of $M_{1}$ was chosen and $y$ was fixed to make the Neutrino Option work, then $\delta, \alpha_{21}, \alpha_{31}, x$ and $M_{2}$ were varied (none of which can spoil the generation of the Higgs potential once $M_{1}$ and $y$ are determined, provided $M_{2}$ does not differ significantly from $M_{1}$ ). We started at the maximum $M_{1}$ which was allowed by the Neutrino Option (which occurs when $y=0^{\circ}$ ) and lowered it in small increments, performing a new parameter scan at each of the successively smaller values of $M_{1}$. This procedure was stopped when the search no longer yielded points in the parameter space where leptogenesis was successful. The lowest value of $M_{1}$ for which leptogenesis was viable was taken as our lower bound. 

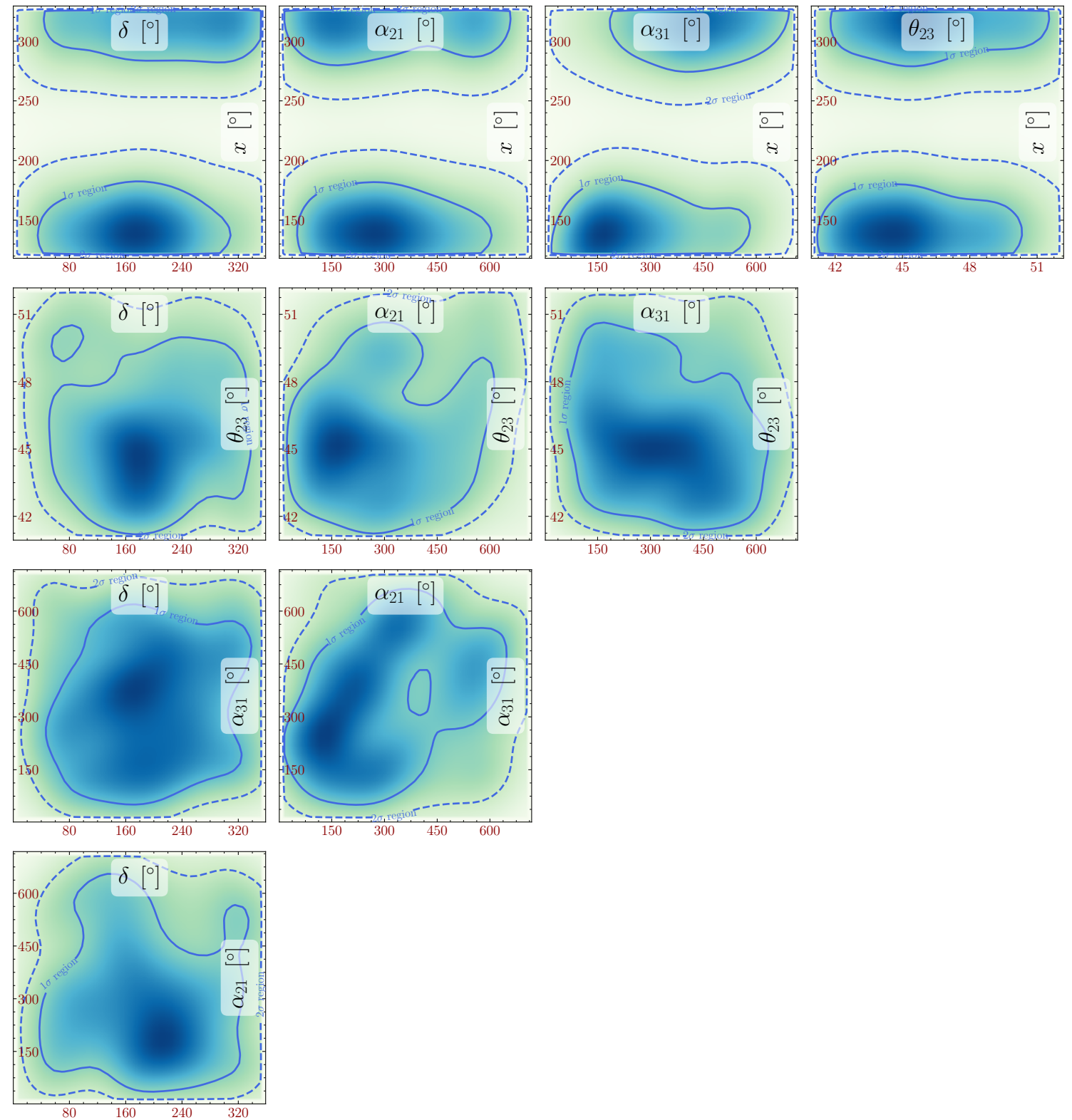

Figure 3. The triangle plot shows regions of the model parameter space compatible with the measured $\eta_{B}$ within $1 \sigma$ and $2 \sigma$ for a normally ordered mass spectrum using resonant leptogenesis with $M=1.2 \times 10^{6} \mathrm{GeV}$. The fixed parameters were set to be $y=190.22^{\circ}, \theta_{12}=33.63^{\circ}$ and $\theta_{13}=8.51^{\circ}$.

At the lowest successful value, for both normal and inverted ordering, we found that the numerical results, as shown in figure 3 and figure 4 (both of which are placed at lower bound on $M$ ), are in broad agreement with the statements made above based on the analytical approximations.

In the inverted ordered case shown in figure 4 , we can see a dependence on $\alpha_{21}$ that is not accounted for in the approximated analytical expressions and that is not present in the normal ordered case of figure 3. The reason is that the suppression factor $e^{-4 y}$ is $\mathcal{O}(100)$ 

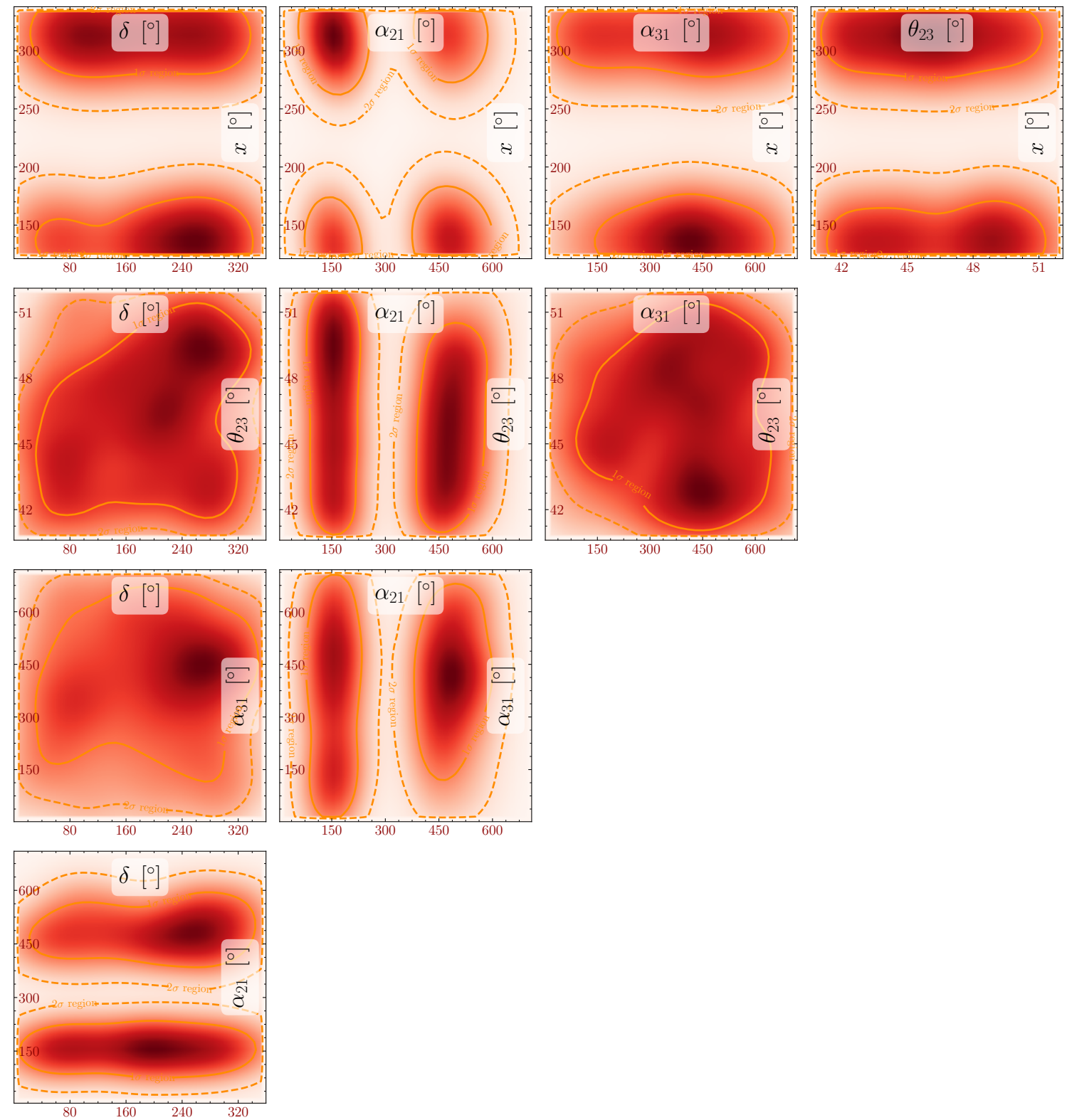

Figure 4. The triangle plot shows regions of the model parameter space compatible with the measured $\eta_{B}$ within one and two $\sigma$ for an inverted ordered mass spectrum using resonant leptogenesis with $M=2.4 \times 10^{6} \mathrm{GeV}$. The fixed parameters were set to be $y=118.21^{\circ}, \theta_{12}=33.63^{\circ}$ and $\theta_{13}=8.51^{\circ}$.

times smaller for inverted ordering, rendering the approximations of eq. (3.4) slightly less accurate than for normal ordering. Terms which were neglected and which depend on $\alpha_{21}$, contribute to a slight dip in the value of $\eta_{B}$ around $\alpha_{21} \sim 300^{\circ}$. The approximate independence from $\delta$ is preserved because terms in $\delta$ are multiplied by the relatively small factor $s_{13}$. Finally, for inverted ordering, independence of $\alpha_{31}$ is exact as it does not appear in the Yukawa matrix when $m_{3}=0$. 
Finally, we note that, at the lower bound for both normal ordering and inverted ordering, the heavy Majorana neutrinos form a pseudo-Dirac pair [18, 19] since, with $x=135^{\circ}$,

$$
Y=U\left(\begin{array}{cc}
0 & 0 \\
-\frac{\sqrt{M_{1} m_{2}}}{\sqrt{2} v}(\cosh y+i \sinh y) & -\frac{\sqrt{M_{2} m_{2}}}{\sqrt{2} v}(\cosh y-i \sinh y) \\
\frac{\sqrt{M_{1} m_{3}}}{\sqrt{2} v}(\cosh y-i \sinh y) & -\frac{\sqrt{M_{2} m_{3}}}{\sqrt{2} v}(\cosh y+i \sinh y)
\end{array}\right)
$$

for which $Y_{1} \approx i Y_{2}$ when $M_{1} \approx M_{2}$. At the lower bound where $\Delta M \ll M$, as the heavy Majorana neutrino mass matrix is diagonal, this condition implies that the CP phases of $N_{1}$ and $N_{2}$ are approximately opposite (see also section 3.3) such that they form a Dirac pair when $M_{1}=M_{2}$. Solutions of this kind may be motivated by assuming an approximate lepton number symmetry [51-64].

\subsection{Upper bound on the heavy Majorana neutrino masses}

The upper bound on $M$ for the Neutrino Option occurs when $y=0^{\circ}$ (eq. (3.2)). In this case one finds for normal ordering

$$
\frac{\epsilon_{\alpha \alpha}^{(1)}}{K_{1} p_{1 \alpha}} \approx \frac{i m_{*}\left(m_{2}-m_{3}\right) \sqrt{m_{2} m_{3}} \cos x \sin x\left(U_{\alpha 2}^{*} U_{\alpha 3}-U_{\alpha 2} U_{\alpha 3}^{*}\right)\left(f_{\mathrm{osc}}+f_{\mathrm{mix}}\right)}{\left(m_{2} \cos ^{2} x+m_{3} \sin ^{2} x\right)\left(m_{3} \cos ^{2} x+m_{2} \sin ^{2} x\right)\left|\sqrt{m_{2}} \cos x U_{\alpha 2}+\sqrt{m_{3}} \sin x U_{\alpha 3}\right|^{2}},
$$

and for inverted ordering

$$
\frac{\epsilon_{\alpha \alpha}^{(1)}}{K_{1} p_{1 \alpha}} \approx \frac{i m_{*}\left(m_{1}-m_{2}\right) \sqrt{m_{1} m_{2}} \cos x \sin x\left(U_{\alpha 1}^{*} U_{\alpha 2}-U_{\alpha 1} U_{\alpha 2}^{*}\right)\left(f_{\mathrm{osc}}+f_{\mathrm{mix}}\right)}{\left(m_{2} \cos ^{2} x+m_{1} \sin ^{2} x\right)\left(m_{1} \cos ^{2} x+m_{2} \sin ^{2} x\right)\left|\sqrt{m_{1}} \cos x U_{\alpha 1}+\sqrt{m_{2}} \sin x U_{\alpha 2}\right|^{2}} .
$$

Unlike for the lower bound where these terms had a maximum value that was largely independent of the PMNS phases, here we find a strong dependence upon these low energy phases and apparently unrestricted enhancement factors. Thus, leptogenesis must be successful at the upper bounds of the Neutrino Option. Combining the lower bounds from requiring the Neutrino Option and leptogenesis to be viable simultaneously with the upper bounds from the Neutrino Option alone results in ${ }^{5}$

$$
\begin{array}{ll}
1.2 \times 10^{6}<M(\mathrm{GeV})<8.8 \times 10^{6} & \text { Normal Ordering } \\
2.4 \times 10^{6}<M(\mathrm{GeV})<7.4 \times 10^{6} & \text { Inverted Ordering } .
\end{array}
$$

In this case the approximate lepton number symmetry need not be so precise as it was for the lower bound.

\subsection{Leptogenesis from purely low energy CP violation in the Neutrino Option}

At the upper bound, for which $y=0^{\circ}$ and therefore $R$ is real, all $\mathrm{CP}$ violation comes from the phases of the PMNS matrix. In this section we consider the possibility that the low

\footnotetext{
${ }^{5}$ The upper bounds quoted here are slightly different from the one reported in ref. [17] owing to the running of light neutrino masses having been neglected in the latter. Numerically the difference amounts to about $\sim 5 \%$ and has therefore limited significance.
} 
energy CP phases $\left(\delta, \alpha_{21}\right.$ and $\left.\alpha_{31}\right)$ provide all of the $\mathrm{CP}$ violation necessary for leptogenesis to produce the observed BAU. In past work it has been shown that these phases are sufficient sources of $\mathrm{CP}$ violation in a mass range $10^{6}<M(\mathrm{GeV})<10^{13}[25,65-72]$ (for a review see, e.g., [73]), where there must be some fine-tuning for heavy Majorana neutrino masses $M \lesssim 10^{9} \mathrm{GeV}$, if they are mildly hierarchical. Here we ask: Is it possible for purely the low energy phases to provide CP violation for successful leptogenesis within the Neutrino Option?

In the type I seesaw, both the light $\left(\nu_{i}\right)$ and the heavy $\left(N_{i}\right)$ neutrino mass states are Majorana and so satisfy the conditions:

$$
\begin{gathered}
C \bar{\nu}_{i}^{T}=\nu_{i}, \\
C \bar{N}_{i}^{T}=N_{i},
\end{gathered}
$$

where $C$ denotes the charge conjugation matrix.

In the case of CP invariance, the Majorana fields $N_{i}(x)$ and $\nu_{i}(x)$ transform as follows under the operation of CP-conjugation (see, e.g., [74]):

$$
\begin{gathered}
U_{C P} N_{i}(x) U_{C P}^{\dagger}=i \rho_{i}^{N} \gamma_{0} N_{i}\left(x^{\prime}\right), \\
U_{C P} \nu_{i}(x) U_{C P}^{\dagger}=i \rho_{i}^{\nu} \gamma_{0} \nu_{i}\left(x^{\prime}\right),
\end{gathered}
$$

where $U_{C P}$ is the CP-conjugation operator, $x^{\prime}$ is the parity-transformed coordinate and $i \rho_{i}^{N}= \pm i$ and $i \rho_{i}^{\nu}= \pm i$ are the $\mathrm{CP}$ parities of the respective Majorana fields. The conditions for $\mathrm{CP}$ invariance impose the following restrictions on the elements of the matrix of neutrino Yukawa couplings:

$$
Y_{\alpha i}^{*}=Y_{\alpha i} \rho_{i}^{N},
$$

where the unphysical phases in the CP transformations of the lepton and Higgs doublets have been set to unity. The CP invariance condition imposed on the PMNS matrix gives the following relation [74]:

$$
U_{\alpha j}^{*}=U_{\alpha j} \rho_{j}^{\nu}, j \in\{1,2,3\}, \alpha \in\{e, \mu, \tau\} .
$$

From the parametrisation of the Yukawa matrix this imposes the following conditions on the elements of the $R$-matrix [67]:

$$
R_{i j}^{*}=R_{i j} \rho_{i}^{N} \rho_{j}^{\nu}, \quad i, j \in\{1,2,3\}
$$

In figure 5 we show a scenario in which the Neutrino Option is satisfied at the upper bound of the normal ordered case and CP violation is provided entirely by the low-energy phases (each phase being zero unless varied). Similarly for inverted ordering of the light neutrinos we have the results depicted in figure 6 . The non-zero values of the baryon asymmetry at the CP conserving values $180^{\circ}$ and $540^{\circ}$ of $\alpha_{21}$ and $\alpha_{31}$ (of $\alpha_{21}$ ) seen in figure 5 (in figure 6) are due to the $\mathrm{CP}$ violating interplay of the CP conserving PMNS and $R$ matrices [67]. This is because there may be a choice of $\rho_{j}^{N}$ that satisfies the condition of eq. (3.10) and $\rho_{i}^{N}$ and $\rho_{j}^{\nu}$ which satisfy the condition of eq. (3.11) but there is no such combination of these phases 

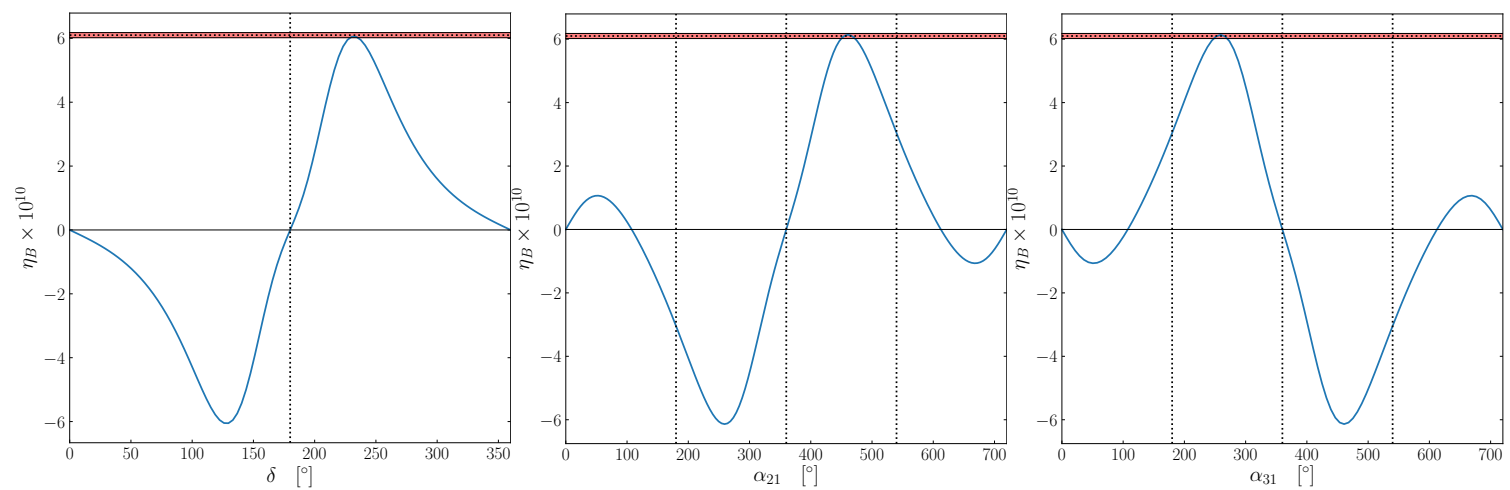

Figure 5. The baryon asymmetry as a function of one of the CPV phases of the PMNS matrix $\delta$, $\alpha_{21}$ and $\alpha_{31}$ (the other two being set to zero), with the varied phase providing all the CP violation in leptogenesis, in the case of neutrino mass spectrum with normal ordering. Vertical dotted lines represent values for which there is a complete $\mathrm{CP}$ symmetry in the neutrino sector. See text for further details.
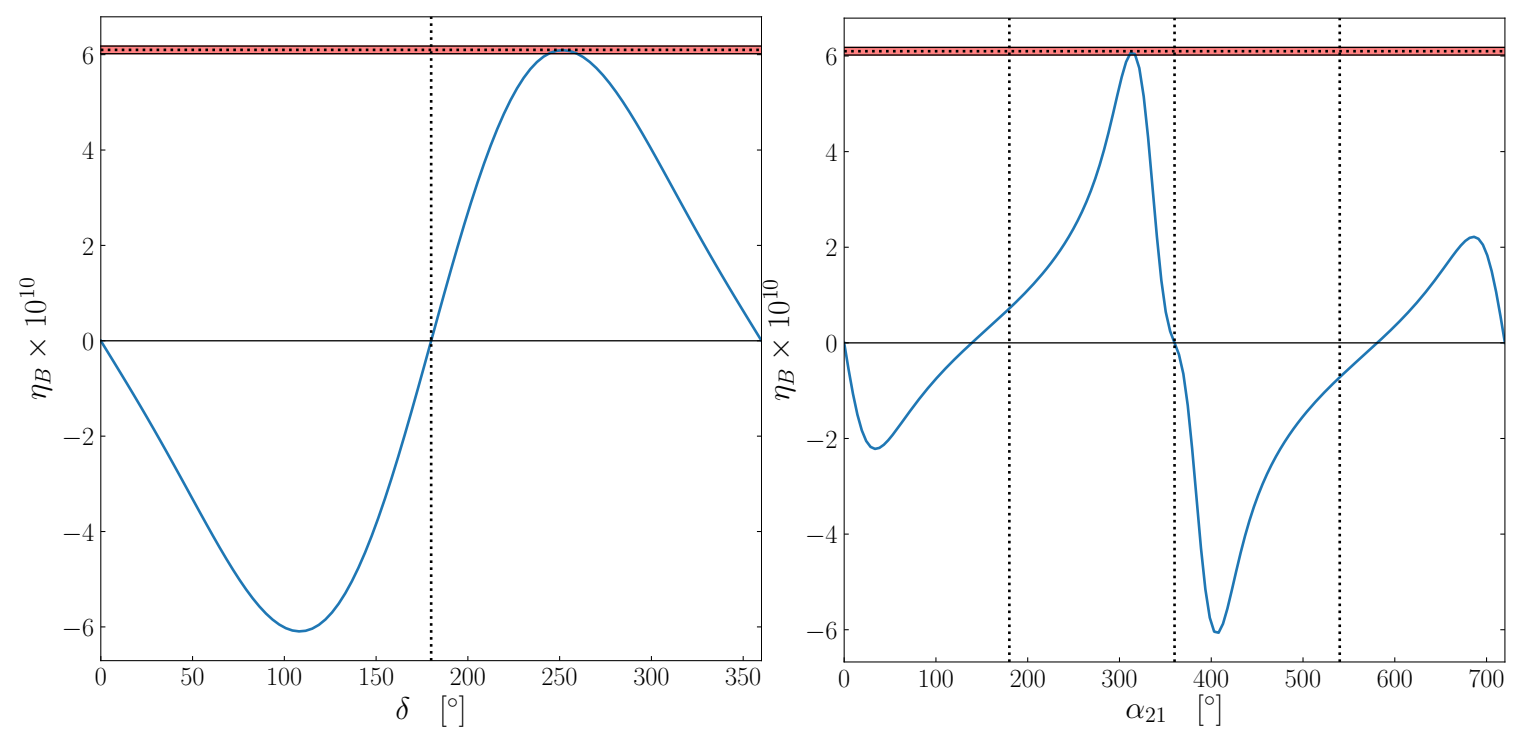

Figure 6. The same as in figure 5 but for neutrino mass spectrum with inverted ordering. The phase $\alpha_{31}$ decouples in this scenario and so a figure with the dependence of the baryon asymmetry on $\alpha_{31}$ is not shown. See text for further details.

that are simultaneously satisfied at these values of $\alpha_{21}$ and $\alpha_{31}$. As a result, the condition of eq. (3.9) on the Yukawa matrix is not fulfilled, leaving it a matrix of complex numbers (with elements not purely real or imaginary) corresponding to the generic CP-violating case.

Although only specific values of the Dirac and Majorana phases are compatible with successful leptogenesis in the context of the Neutrino Option, it is important to emphasise that only the Dirac is measurable at neutrino oscillation experiments. In the scenario the Majorana phases are the sole CP-violating phases, a CP-conserving measurement of $\delta$ would still be compatible with producing the BAU in this framework. Nonetheless, for each scenario (be it sole CP-violation from $\delta, \alpha_{21}$ or $\alpha_{31}$ ) a prediction for the value of the effective Majorana mass, $\left|m_{e e}\right|$, and the corresponding half-life of neutrinoless double beta 
decay, can be calculated. In principle, assuming precise knowledge of the neutrino masses and an accurate evaluation of nuclear matrix elements, this prediction can be tested by neutrinoless double beta decay experiments which will probe the inverted ordering regime in the near future (see ref. [75] for further discussion on this matter).

\section{Conclusions}

The aim of this paper is to examine resonant leptogenesis in the context of the Neutrino Option thereby taking one further step in constructing a self-consistent theory which simultaneously explains light neutrino masses, the predominance of matter over anti-matter, and the electroweak scale.

We found that the viable parameter space which can satisfy the Neutrino Option and leptogenesis are in the ranges $1.2 \times 10^{6}<M(\mathrm{GeV})<8.8 \times 10^{6}$ and $2.4 \times 10^{6}<M(\mathrm{GeV})<$ $7.4 \times 10^{6}$ for normal and inverted ordering respectively, with successful leptogenesis requiring a pseudo-Dirac pair with masses such that $\Delta M / M \equiv\left(M_{2}-M_{1}\right) / M \sim 10^{-8}$. Interestingly, viable solutions for Neutrino Option leptogenesis allows for $\theta_{23}$ be to in the lower or upper octant (at the $2 \sigma$ level) however for normal ordering there is a slight preference for solutions in the upper octant. In particular, we found that, generally, there is only a weak dependence on the low energy phases of the PMNS matrix $\delta, \alpha_{21}$ and $\alpha_{31}$ at the lower bounds on the viable mass range for both normal and inverted ordering (see figure 3 and figure 4). The minor exception to this is $\alpha_{21}$ in the case of inverted ordering which must be approximately in the range $\left[90^{\circ}, 200^{\circ}\right]$ or $\left[360^{\circ}, 600^{\circ}\right]$ for $1 \sigma$ agreement with $\eta_{B_{\mathrm{CMB}}}$.

We have further shown successful leptogenesis within the framework of the Neutrino Option scenario is possible when the requisite $\mathrm{CP}$ violation in leptogenesis is provided exclusively by the Dirac or Majorana low-energy CP violation phases of the PMNS matrix. This is possible only at the upper bound of the viable mass range and provides a stark contrast with leptogenesis at the lower bounds where the low-energy PMNS phases were largely irrelevant.

\section{Acknowledgments}

We would like to thank Bhupal Dev and Peter Millington for helpful discussions regarding resonant leptogenesis. K.M. and S.P. acknowledge the (partial) support from the European Research Council under the European Union Seventh Framework Programme (FP/20072013)/ERC Grant NuMass agreement n. [617143]. S.P. would like to acknowledge partial support from the Wolfson Foundation and the Royal Society, and also thanks SISSA for support and hospitality during part of this work. S.P. and S.T.P. acknowledge partial support from the European Unions Horizon 2020 research and innovation programme under the Marie Sklodowska Curie grant agreements No 690575 (RISE InvisiblesPlus) and No. 674896 (ITN ELUSIVE). The work of S.T.P. was supported in part by the INFN program on Theoretical Astroparticle Physics (TASP) and by the World Premier International Research Center Initiative (WPI Initiative), MEXT, Japan. This manuscript has been authored by Fermi Research Alliance, LLC under Contract No. DE-AC02-07CH11359 with the U.S. Department of Energy, Office of Science, Office of High Energy Physics. 
Open Access. This article is distributed under the terms of the Creative Commons Attribution License (CC-BY 4.0), which permits any use, distribution and reproduction in any medium, provided the original author(s) and source are credited.

\section{References}

[1] P. Minkowski, $\mu \rightarrow$ er at a Rate of One Out of $10^{9}$ Muon Decays?, Phys. Lett. 67B (1977) 421 [INSPIRE].

[2] T. Yanagida, Horizontal gauge symmetry and masses of neutrinos, Conf. Proc. C 7902131 (1979) 95 [INSPIRE].

[3] M. Gell-Mann, P. Ramond and R. Slansky, Complex Spinors and Unified Theories, Conf. Proc. C 790927 (1979) 315 [arXiv:1306.4669] [InSPIRE].

[4] R.N. Mohapatra and G. Senjanović, Neutrino Mass and Spontaneous Parity Nonconservation, Phys. Rev. Lett. 44 (1980) 912 [INSPIRE].

[5] M. Fukugita and T. Yanagida, Baryogenesis Without Grand Unification, Phys. Lett. B 174 (1986) 45 [INSPIRE].

[6] W. Buchmüller and M. Plümacher, Baryon asymmetry and neutrino mixing, Phys. Lett. B 389 (1996) 73 [hep-ph/9608308] [INSPIRE].

[7] W. Buchmüller, P. Di Bari and M. Plümacher, Leptogenesis for pedestrians, Annals Phys. 315 (2005) 305 [hep-ph/0401240] [INSPIRE].

[8] W. Buchmüller, R.D. Peccei and T. Yanagida, Leptogenesis as the origin of matter, Ann. Rev. Nucl. Part. Sci. 55 (2005) 311 [hep-ph/0502169] [INSPIRE].

[9] A. Pilaftsis, CP violation and baryogenesis due to heavy Majorana neutrinos, Phys. Rev. D 56 (1997) 5431 [hep-ph/9707235] [INSPIRE].

[10] A.D. Sakharov, Violation of CP Invariance, $C$ asymmetry and baryon asymmetry of the universe, Pisma Zh. Eksp. Teor. Fiz. 5 (1967) 32 [INSPIRE].

[11] F. Vissani, Do experiments suggest a hierarchy problem?, Phys. Rev. D 57 (1998) 7027 [hep-ph/9709409] [INSPIRE].

[12] J.D. Clarke, R. Foot and R.R. Volkas, Electroweak naturalness in the three-flavor type-I seesaw model and implications for leptogenesis, Phys. Rev. D 91 (2015) 073009 [arXiv: 1502.01352] [INSPIRE].

[13] S. Davidson and A. Ibarra, A Lower bound on the right-handed neutrino mass from leptogenesis, Phys. Lett. B 535 (2002) 25 [hep-ph/0202239] [INSPIRE].

[14] W. Buchmüller, P. Di Bari and M. Plümacher, Cosmic microwave background, matter-antimatter asymmetry and neutrino masses, Nucl. Phys. B 643 (2002) 367 [Erratum ibid. B 793 (2008) 362] [hep-ph/0205349] [INSPIRE].

[15] J.R. Ellis and M. Raidal, Leptogenesis and the violation of lepton number and CP at low-energies, Nucl. Phys. B 643 (2002) 229 [hep-ph/0206174] [INSPIRE].

[16] I. Brivio and M. Trott, Radiatively Generating the Higgs Potential and Electroweak Scale via the Seesaw Mechanism, Phys. Rev. Lett. 119 (2017) 141801 [arXiv:1703.10924] [InSPIRE].

[17] I. Brivio and M. Trott, Examining the neutrino option, JHEP 02 (2019) 107 [arXiv: 1809.03450] [INSPIRE]. 
[18] L. Wolfenstein, Different Varieties of Massive Dirac Neutrinos, Nucl. Phys. B 186 (1981) 147 [INSPIRE].

[19] S.T. Petcov, On PseudoDirac Neutrinos, Neutrino Oscillations and Neutrinoless Double beta Decay, Phys. Lett. 110B (1982) 245 [inSPIRE].

[20] Particle Data Group collaboration, Review of Particle Physics, Phys. Rev. D 98 (2018) 030001 [INSPIRE].

[21] S.M. Bilenky, J. Hosek and S.T. Petcov, On Oscillations of Neutrinos with Dirac and Majorana Masses, Phys. Lett. 94B (1980) 495 [inSPIRE].

[22] I. Esteban, M.C. Gonzalez-Garcia, A. Hernandez-Cabezudo, M. Maltoni and T. Schwetz, Global analysis of three-flavour neutrino oscillations: synergies and tensions in the determination of $\theta_{23}, \delta_{C P}$ and the mass ordering, JHEP 01 (2019) 106 [arXiv:1811.05487] [INSPIRE].

[23] J.A. Casas and A. Ibarra, Oscillating neutrinos and $\mu \rightarrow e, \gamma$, Nucl. Phys. B 618 (2001) 171 [hep-ph/0103065] [INSPIRE].

[24] Planck collaboration, Planck 2018 results. VI. Cosmological parameters, arXiv:1807.06209 [INSPIRE].

[25] E. Molinaro and S.T. Petcov, The Interplay Between the 'Low' and 'High' Energy CP-Violation in Leptogenesis, Eur. Phys. J. C 61 (2009) 93 [arXiv:0803.4120] [inSPIRE].

[26] D. Buttazzo et al., Investigating the near-criticality of the Higgs boson, JHEP 12 (2013) 089 [arXiv: 1307.3536] [INSPIRE].

[27] Particle Data Group collaboration, Review of Particle Physics, Chin. Phys. C 40 (2016) 100001 [INSPIRE].

[28] Planck collaboration, Planck 2015 results. XIII. Cosmological parameters, Astron. Astrophys. 594 (2016) A13 [arXiv:1502.01589] [INSPIRE].

[29] S. Blanchet, P. Di Bari, D.A. Jones and L. Marzola, Leptogenesis with heavy neutrino flavours: from density matrix to Boltzmann equations, JCAP 01 (2013) 041 [arXiv: 1112.4528] [INSPIRE].

[30] A. Pilaftsis and T.E.J. Underwood, Resonant leptogenesis, Nucl. Phys. B 692 (2004) 303 [hep-ph/0309342] [INSPIRE].

[31] P.S. Bhupal Dev, P. Millington, A. Pilaftsis and D. Teresi, Flavour Covariant Transport Equations: an Application to Resonant Leptogenesis, Nucl. Phys. B 886 (2014) 569 [arXiv: 1404.1003] [INSPIRE].

[32] P.S. Bhupal Dev, P. Millington, A. Pilaftsis and D. Teresi, Kadanoff-Baym approach to flavour mixing and oscillations in resonant leptogenesis, Nucl. Phys. B 891 (2015) 128 [arXiv: 1410.6434] [INSPIRE].

[33] G. Bambhaniya, P.S. Bhupal Dev, S. Goswami, S. Khan and W. Rodejohann, Naturalness, Vacuum Stability and Leptogenesis in the Minimal Seesaw Model, Phys. Rev. D 95 (2017) 095016 [arXiv: 1611.03827] [INSPIRE].

[34] K. Moffat, S. Pascoli, S.T. Petcov, H. Schulz and J. Turner, Three-flavored nonresonant leptogenesis at intermediate scales, Phys. Rev. D 98 (2018) 015036 [arXiv:1804.05066] [INSPIRE]. 
[35] S. Blanchet and P. Di Bari, New aspects of leptogenesis bounds, Nucl. Phys. B 807 (2009) 155 [arXiv: 0807.0743] [INSPIRE].

[36] S. Antusch, S. Blanchet, M. Blennow and E. Fernandez-Martinez, Non-unitary Leptonic Mixing and Leptogenesis, JHEP 01 (2010) 017 [arXiv:0910.5957] [INSPIRE].

[37] A. Pilaftsis and T.E.J. Underwood, Electroweak-scale resonant leptogenesis, Phys. Rev. D 72 (2005) 113001 [hep-ph/0506107] [InSPIRE].

[38] T. Hambye, Leptogenesis at the TeV scale, Nucl. Phys. B 633 (2002) 171 [hep-ph/0111089] [INSPIRE].

[39] T. Hambye, J. March-Russell and S.M. West, TeV scale resonant leptogenesis from supersymmetry breaking, JHEP 07 (2004) 070 [hep-ph/0403183] [INSPIRE].

[40] V. Cirigliano, G. Isidori and V. Porretti, CP violation and Leptogenesis in models with Minimal Lepton Flavour Violation, Nucl. Phys. B 763 (2007) 228 [hep-ph/0607068] [INSPIRE].

[41] Z.-z. Xing and S. Zhou, Tri-bimaximal Neutrino Mixing and Flavor-dependent Resonant Leptogenesis, Phys. Lett. B 653 (2007) 278 [hep-ph/0607302] [INSPIRE].

[42] G.C. Branco, A.J. Buras, S. Jager, S. Uhlig and A. Weiler, Another look at minimal lepton flavour violation, $l_{i} \rightarrow l_{j \gamma}$, leptogenesis and the ratio $M_{\nu} / \Lambda_{L F V}$, JHEP 09 (2007) 004 [hep-ph/0609067] [INSPIRE].

[43] E.J. Chun and K. Turzynski, Quasi-degenerate neutrinos and leptogenesis from $L_{\mu}-L_{\tau}$, Phys. Rev. D 76 (2007) 053008 [hep-ph/0703070] [InSPIRE].

[44] T. Kitabayashi, Remark on the minimal seesaw model and leptogenesis with tri/bi-maximal mixing, Phys. Rev. D 76 (2007) 033002 [hep-ph/0703303] [INSPIRE].

[45] J. Lopez-Pavon, E. Molinaro and S.T. Petcov, Radiative Corrections to Light Neutrino Masses in Low Scale Type I Seesaw Scenarios and Neutrinoless Double Beta Decay, JHEP 11 (2015) 030 [arXiv: 1506.05296] [INSPIRE].

[46] J.A. Casas, J.R. Espinosa, A. Ibarra and I. Navarro, General RG equations for physical neutrino parameters and their phenomenological implications, Nucl. Phys. B 573 (2000) 652 [hep-ph/9910420] [INSPIRE].

[47] S. Antusch, J. Kersten, M. Lindner and M. Ratz, Running neutrino masses, mixings and CP phases: Analytical results and phenomenological consequences, Nucl. Phys. B 674 (2003) 401 [hep-ph/0305273] [INSPIRE].

[48] F. Feroz and M.P. Hobson, Multimodal nested sampling: an efficient and robust alternative to MCMC methods for astronomical data analysis, Mon. Not. Roy. Astron. Soc. 384 (2008) 449 [arXiv:0704.3704] [INSPIRE].

[49] F. Feroz, M.P. Hobson and M. Bridges, MultiNest: an efficient and robust Bayesian inference tool for cosmology and particle physics, Mon. Not. Roy. Astron. Soc. 398 (2009) 1601 [arXiv:0809.3437] [INSPIRE].

[50] F. Feroz, M.P. Hobson, E. Cameron and A.N. Pettitt, Importance Nested Sampling and the MultiNest Algorithm, arXiv:1306.2144 [INSPIRE].

[51] R.N. Mohapatra, Mechanism for Understanding Small Neutrino Mass in Superstring Theories, Phys. Rev. Lett. 56 (1986) 561 [InSPIRE]. 
[52] R.N. Mohapatra and J.W.F. Valle, Neutrino Mass and Baryon Number Nonconservation in Superstring Models, Phys. Rev. D 34 (1986) 1642 [InSPIRE].

[53] J. Bernabeu, A. Santamaria, J. Vidal, A. Mendez and J.W.F. Valle, Lepton Flavor Nonconservation at High-Energies in a Superstring Inspired Standard Model, Phys. Lett. B 187 (1987) 303 [INSPIRE].

[54] A. Pilaftsis, Radiatively induced neutrino masses and large Higgs neutrino couplings in the standard model with Majorana fields, Z. Phys. C 55 (1992) 275 [hep-ph/9901206] [InSPIRE].

[55] A. Ilakovac and A. Pilaftsis, Flavor violating charged lepton decays in seesaw-type models, Nucl. Phys. B 437 (1995) 491 [hep-ph/9403398] [INSPIRE].

[56] E.K. Akhmedov, M. Lindner, E. Schnapka and J.W.F. Valle, Left-right symmetry breaking in NJLS approach, Phys. Lett. B 368 (1996) 270 [hep-ph/9507275] [INSPIRE].

[57] E.K. Akhmedov, M. Lindner, E. Schnapka and J.W.F. Valle, Dynamical left-right symmetry breaking, Phys. Rev. D 53 (1996) 2752 [hep-ph/9509255] [INSPIRE].

[58] A. Abada, G. Bhattacharyya, D. Das and C. Weiland, A possible connection between neutrino mass generation and the lightness of a NMSSM pseudoscalar, Phys. Lett. B 700 (2011) 351 [arXiv:1011.5037] [INSPIRE].

[59] A. Abada, D. Das and C. Weiland, Enhanced Higgs Mediated Lepton Flavour Violating Processes in the Supersymmetric Inverse Seesaw Model, JHEP 03 (2012) 100 [arXiv:1111.5836] [INSPIRE].

[60] R. Alonso, E. Fernandez Martinez, M.B. Gavela, B. Grinstein, L. Merlo and P. Quilez, Gauged Lepton Flavour, JHEP 12 (2016) 119 [arXiv:1609.05902] [INSPIRE].

[61] M.B. Gavela, T. Hambye, D. Hernandez and P. Hernández, Minimal Flavour Seesaw Models, JHEP 09 (2009) 038 [arXiv: 0906.1461] [INSPIRE].

[62] A.G. Dias, C.A. de S. Pires and P.S.R. da Silva, How the Inverse See-Saw Mechanism Can Reveal Itself Natural, Canonical and Independent of the Right-Handed Neutrino Mass, Phys. Rev. D 84 (2011) 053011 [arXiv: 1107.0739] [INSPIRE].

[63] F. Bazzocchi, Minimal Dynamical Inverse See Saw, Phys. Rev. D 83 (2011) 093009 [arXiv: 1011.6299] [INSPIRE].

[64] E. Ma, Radiative inverse seesaw mechanism for nonzero neutrino mass, Phys. Rev. D 80 (2009) 013013 [arXiv:0904.4450] [INSPIRE].

[65] K. Moffat, S. Pascoli, S.T. Petcov and J. Turner, Leptogenesis from Low Energy CP Violation, JHEP 03 (2019) 034 [arXiv: 1809.08251] [INSPIRE].

[66] S. Pascoli, S.T. Petcov and A. Riotto, Connecting low energy leptonic CP-violation to leptogenesis, Phys. Rev. D 75 (2007) 083511 [hep-ph/0609125] [INSPIRE].

[67] S. Pascoli, S.T. Petcov and A. Riotto, Leptogenesis and Low Energy CP-violation in Neutrino Physics, Nucl. Phys. B 774 (2007) 1 [hep-ph/0611338] [INSPIRE].

[68] S. Blanchet and P. Di Bari, Flavor effects on leptogenesis predictions, JCAP 03 (2007) 018 [hep-ph/0607330] [INSPIRE].

[69] G.C. Branco, R. Gonzalez Felipe and F.R. Joaquim, A New bridge between leptonic CP-violation and leptogenesis, Phys. Lett. B 645 (2007) 432 [hep-ph/0609297] [INSPIRE].

[70] A. Anisimov, S. Blanchet and P. Di Bari, Viability of Dirac phase leptogenesis, JCAP 04 (2008) 033 [arXiv:0707.3024] [INSPIRE]. 
[71] E. Molinaro and S.T. Petcov, A Case of Subdominant/Suppressed 'High Energy' Contribution to the Baryon Asymmetry of the Universe in Flavoured Leptogenesis, Phys. Lett. B 671 (2009) 60 [arXiv:0808.3534] [INSPIRE].

[72] M.J. Dolan, T.P. Dutka and R.R. Volkas, Dirac-Phase Thermal Leptogenesis in the extended Type-I Seesaw Model, JCAP 06 (2018) 012 [arXiv: 1802.08373] [INSPIRE].

[73] C. Hagedorn, R.N. Mohapatra, E. Molinaro, C.C. Nishi and S.T. Petcov, CP Violation in the Lepton Sector and Implications for Leptogenesis, Int. J. Mod. Phys. A 33 (2018) 1842006 [arXiv: 1711.02866] [INSPIRE].

[74] S.M. Bilenky and S.T. Petcov, Massive Neutrinos and Neutrino Oscillations, Rev. Mod. Phys. 59 (1987) 671 [Erratum ibid. 61 (1989) 169] [INSPIRE].

[75] S. Pascoli, S.T. Petcov and T. Schwetz, The Absolute neutrino mass scale, neutrino mass spectrum, Majorana CP-violation and neutrinoless double-beta decay, Nucl. Phys. B 734 (2006) 24 [hep-ph/0505226] [INSPIRE]. 